\title{
A NEW CLASS OF GENERALIZED POLYNOMIALS ASSOCIATED WITH HERMITE AND POLY-BERNOULLI POLYNOMIALS
}

\author{
M. A. PATHAN AND WASEEM A. KHAN
}

Received 22 May, 2015

\begin{abstract}
In this paper, we introduce a new class of generalized polynomials associated with the modified Milne-Thomson's polynomials $\Phi_{n}^{(\alpha)}(x, v)$ of degree $n$ and order $\alpha$ introduced by Dere and Simsek. The concepts of poly-Bernoulli numbers, poly-Bernoulli polynomials, HermiteBernoulli polynomials and generalized Hermite-Bernoulli polynomials are generalized to polynomials of three positive real parameters. Numerous properties of these polynomials and some relations are established. Some implicit summation formulae and general symmetry identities are derived by using different analytical means and applying generating functions. These results extend some known summations and identities of generalized poly-Bernoulli numbers and polynomials.
\end{abstract}

2010 Mathematics Subject Classification: 11B73; 11B68; 33C45

Keywords: Hermite polynomials, Bernoulli polynomials, poly-Bernoulli polynomials, Hermitepoly-Bernoulli polynomials, summation formulae, symmetric identities

\section{INTRODUCTION}

Kaneko [6] introduced and studied poly-Bernoulli numbers which generalize the classical Bernoulli numbers. poly-Bernoulli numbers $B_{n}^{(k)}$ with $k \in \mathbb{Z}$ and $n \in \mathbb{N}$, appear in the following power series:

$$
\frac{\operatorname{Li}_{k}\left(1-e^{-t}\right)}{1-e^{-t}}=\sum_{n=0}^{\infty} B_{n}^{(k)} \frac{t^{n}}{n !}
$$

where

$$
\operatorname{Li}_{k}(z)=\sum_{m=1}^{\infty} \frac{z^{m}}{m^{k}},|z|<1
$$

and

$$
\operatorname{Li}_{1}(z)=-\ln (1-z), \operatorname{Li}_{0}(z)=\frac{z}{1-z}, \operatorname{Li}_{-1}(z)=\frac{z}{(1-z)^{2}}, \ldots
$$


Moreover when $k \geq 1$, the left hand side of (1.1) can be written in the form of iterated integrals

$$
e^{t} \frac{1}{e^{t}-1} \int_{0}^{t} \frac{1}{e^{t}-1} \cdots \int_{0}^{t} \frac{1}{e^{t}-1} \int_{0}^{t} \frac{t}{e^{t}-1} d t d t \cdots d t=\sum_{n=0}^{\infty} B_{n}^{(k)} \frac{t^{n}}{n !}
$$

Obviously

$$
B_{n}^{(1)}=B_{n} .
$$

Recently, Jolany et al. [4,5] generalized the concept of poly-Bernoulli polynomials defined as follows.

Let $a, b, c>0$ and $a \neq b$. The generalized poly-Bernoulli numbers $B_{n}^{(k)}(a, b)$, the generalized poly-Bernoulli polynomials $B_{n}^{(k)}(x ; a, b)$ and the generalized polynomials $B_{n}^{(k)}(x ; a, b, c)$ are appeared in the following series respectively.

$$
\begin{aligned}
\frac{\operatorname{Li}_{k}\left(1-(a b)^{-t}\right)}{b^{t}-a^{-t}} & =\sum_{n=0}^{\infty} B_{n}^{(k)}(a, b) \frac{t^{n}}{n !},|t|<\frac{2 \pi}{|\ln a+\ln b|}, \\
\frac{\operatorname{Li}_{k}\left(1-(a b)^{-t}\right)}{b^{t}-a^{-t}} e^{x t} & =\sum_{n=0}^{\infty} B_{n}^{(k)}(x, a, b) \frac{t^{n}}{n !},|t|<\frac{2 \pi}{|\ln a+\ln b|}, \\
\frac{\operatorname{Li}_{k}\left(1-(a b)^{-t}\right)}{b^{t}-a^{-t}} c^{x t} & =\sum_{n=0}^{\infty} B_{n}^{(k)}(x, a, b, c) \frac{t^{n}}{n !},|t|<\frac{2 \pi}{|\ln a+\ln b|} .
\end{aligned}
$$

Dere and Simsek [3] modified the Milne-Thomson's polynomials $\Phi_{n}^{(\alpha)}(x)$ (see for detail [11]) as $\Phi_{n}^{(\alpha)}(x, v)$ of degree $n$ and order $\alpha$ by the means of the following generating function:

$$
g_{1}(t, x ; \alpha, v)=f(t, \alpha) e^{x t+h(t, v)}=\sum_{n=0}^{\infty} \Phi_{n}^{(\alpha)}(x, v) \frac{t^{n}}{n !},
$$

where $f(t, \alpha)$ is a function of $t$ and integer $\alpha$. Note that $\Phi_{n}^{(\alpha)}(x, 0)=\Phi_{n}^{(\alpha)}(x)$ (c.f. [11]).

On setting $f(t, \alpha)=\frac{\mathrm{Li}_{k}\left(1-e^{-t}\right)}{1-e^{-t}}$ in (1.5), we obtain the following polynomials given by the generating function:

$$
g_{2}(t, x ; k, v)=\frac{\operatorname{Li}_{k}\left(1-e^{-t}\right)}{1-e^{-t}} e^{x t+h(t, v)}=\sum_{n=0}^{\infty} \frac{B_{n}^{(k)}(x, v) t^{n}}{n !}
$$

Observe that the polynomials $B_{n}^{(k)}(x, v)$ are related to not only Bernoulli polynomials but also the Hermite polynomials. For example, if $h(t, 0)=0$ in (1.6), we have

$$
B_{n}^{(k)}(x, 0)=B_{n}^{(k)}(x)
$$


where $B_{n}^{(k)}(x)$ denotes the poly-Bernoulli polynomials of higher order which is defined by means of the following generating function:

$$
F_{B}(t, x ; k)=\frac{\operatorname{Li}_{k}\left(1-e^{-t}\right)}{1-e^{-t}} e^{x t}=\sum_{n=0}^{\infty} B_{n}^{(k)}(x) \frac{t^{n}}{n !} .
$$

One can easily see that

$$
B_{n}^{(k)}(0,0)=B_{n}^{(k)}, B_{n}^{(k)}(x)=1+x
$$

and

$$
B_{n}^{(k)}(x)=B_{n}^{(k)}\left(e^{x+1}, e^{x}\right),
$$

where $B_{n}^{(k)}$ are generalized poly-Bernoulli numbers. For more information about poly-Bernoulli numbers and poly-Bernoulli polynomials, we refer to [4-8].

In [10], Luo et al. gave the following definition of the generalized Bernoulli polynomials, which generalize the concepts stated above.

Let $a, b>0$ and $a \neq b$. The generalized Bernoulli polynomials $B_{n}(x ; a, b, c)$ for nonnegative integer $\mathrm{n}$ are defined by

$$
\Phi(x, t ; a, b, c)=\frac{t}{a^{t}-b^{t}} c^{x t}=\sum_{n=0}^{\infty} B_{n}(x ; a, b, c) \frac{t^{n}}{n !}, \quad|t|<2 \pi
$$

Let $x, y \in \mathbb{R}$, the generalized Hermite-Bernoulli polynomials of two variables given by means of the following generating function (see [14]):

$$
\left(\frac{t}{a^{t}-b^{t}}\right)^{\alpha} c^{x t+y t^{2}}=\sum_{n=0}^{\infty}{ }_{H} B_{n}^{(\alpha)}(x, y ; a, b, c) \frac{t^{n}}{n !}, \quad|t|<\frac{2 \pi}{|\ln a+\ln b|},
$$

which is essentially a generalization of Bernoulli numbers, Bernoulli polynomials, Hermite polynomials and Hermite-Bernoulli polynomials ${ }_{H} B_{n}(x, y)$, generalized Hermite-Bernoulli polynomials ${ }_{H} B_{n}^{(\alpha)}(x, y)$ introduced by Pathan and Khan [15] and Dattoli et al. [2, p.386(1.6)] in the form:

$$
\left(\frac{t}{e^{t}-1}\right)^{\alpha} e^{x t+y t^{2}}=\sum_{n=0}^{\infty}{ }_{H} B_{n}^{(\alpha)}(x, y) \frac{t^{n}}{n !},
$$

and

$$
\left(\frac{t}{e^{t}-1}\right) e^{x t+y t^{2}}=\sum_{n=0}^{\infty}{ }_{H} B_{n}(x, y) \frac{t^{n}}{n !} .
$$

Let $c>0$. The generalized 2-variable 1-parameter Hermite Kampé de Fériet $H_{n}(x, y, c)$ polynomials for nonnegative integer $n$ are defined by

$$
c^{x t+y t^{2}}=\sum_{n=0}^{\infty} H_{n}(x, y, c) \frac{t^{n}}{n !} .
$$


This is an extended 2-variable Hermite Kamp'e de Feriet polynomials $H_{n}(x, y)$ (see [1]) defined by

$$
e^{x t+y t^{2}}=\sum_{n=0}^{\infty} H_{n}(x, y) \frac{t^{n}}{n !}
$$

Note that

$$
H_{n}(x, y, e)=H_{n}(x, y),
$$

and the definition (1.13) yields the relationship

$$
H_{n}(x, y, c)=\sum_{j=0}^{\left[\frac{n}{2}\right]}\left(\begin{array}{c}
n \\
j
\end{array}\right)(\ln c)^{n-j} x^{n-2 j} y^{j} .
$$

In this note, we first give definitions of the generalized poly-Bernoulli polynomials $B_{n}^{(k)}(x ; a, b . c)$, which generalize the concepts stated above and then research their basic properties and relationships with poly-Bernoulli numbers $B_{n}^{(k)}(a, b)$, poly-Bernoulli polynomials $B_{n}^{(k)}(x)$ and the generalized poly-Bernoulli polynomials $B_{n}^{(k)}(x ; a, b, c)$ of Joalny et al., Hermite-Bernoulli polynomials ${ }_{H} B_{n}(x, y)$ of Dattoli et al. and ${ }_{H} B_{n}^{(\alpha)}(x, y)$ of Pathan and Khan. The remainder of this paper is organized as follows. We modify generating functions for the Milne-Thomson's polynomials and derive some identities related to Hermite polynomials, poly-Bernoulli polynomials and power sums. Some implicit summation formulae and general symmetry identities are derived by using different analytical means and applying generating functions. These results extend some known summations and identities of generalized Hermite-poly-Bernoulli polynomials, degenerate Hermite poly-Bernoulli studied by Khan [7-9].

\section{DEFINITION AND PROPERTIES OF THE GENERALIZED HERMite POLY-BERNOULLI POLYNOMIALS ${ }_{H} B_{n}^{(k)}(x, y ; a, b, c)$}

In the modified Milne Thomson's polynomials due to Dere and Simsek [3,11] defined by (1.5), if we set $f(t, \alpha)=\frac{\mathrm{Li}_{k}\left(1-(a b)^{-t}\right)}{b^{t}-a^{-t}}$, we obtain the following generalized polynomials $B_{n}^{(k)}(x, v ; a, b, c)$.

Definition 1. Let $a, b, c>0$ and $a \neq b$. The generalized poly-Bernoulli polynomials $B_{n}^{(k)}(x, \mathrm{v} ; a, b, c)$ are defined by

$$
\begin{aligned}
G_{1}(t, x ; \alpha, a, b, v)= & \frac{\operatorname{Li}_{k}\left(1-(a b)^{-t}\right)}{b^{t}-a^{-t}} c^{x t+h(t, v)}=\sum_{n=0}^{\infty} B_{n}^{(k)}(x, v ; a, b, c) \frac{t^{n}}{n !}, \\
& (|t|<2 \pi /(|\ln a+\ln b|), x \in \mathbb{R}) .
\end{aligned}
$$

On setting $h(t, v)=h(t, y)=y t^{2},(2.1)$ reduces to 
Definition 2. Let $a, b, c>0$ and $a \neq b$. The generalized Hermite poly-Bernoulli polynomials ${ }_{H} B_{n}^{(k)}(x, y ; a, b, c)$ are defined by

$$
\begin{aligned}
G_{2}(t, x, y ; k, a, b, c)= & \frac{\mathrm{Li}_{k}\left(1-(a b)^{-t}\right)}{b^{t}-a^{-t}} c^{x t+y t^{2}}=\sum_{n=0}^{\infty}{ }_{H} B_{n}^{(k)}(x, y ; a, b, c) \frac{t^{n}}{n !}, \\
& (|t|<2 \pi /(|\ln a+\ln b|), x, y \in \mathbb{R}),
\end{aligned}
$$

whereas for $x=0$ gives

$$
{ }_{H} B_{n}^{(k)}(0, y ; a, b, c)=\sum_{m=0}^{\left[\frac{n}{2}\right]} \frac{n !}{m !(n-2 m) !}(\ln c)^{m} B_{n-2 m}^{(k)}(a, b) y^{m} .
$$

Another special case of (2.2), for $y=0$ leads to the extension of the generalized poly-Bernoulli numbers $B_{n}^{(k)}(a, b)$ defined by (1.2) in the form.

Definition 3. Let $a, b, c>0$ and $a \neq b$. The generalized poly-Bernoulli polynomials $B_{n}^{(k)}(x ; a, b, c)$ are defined by

$$
\begin{gathered}
\Phi(t ; k, a, b)=\frac{\mathrm{Li}_{k}\left(1-(a b)^{-t}\right)}{b^{t}-a^{-t}} c^{x t}=\sum_{n=0}^{\infty} B_{n}^{(k)}(x ; a, b, c) \frac{t^{n}}{n !}, \\
(|t|<2 \pi /(|\ln a+\ln b|), x \in \mathbb{R}) .
\end{gathered}
$$

Letting $c=e$, equation (2.2) reduces to

Definition 4. Let $a, b>0$ and $a \neq b$. The generalized Hermite poly-Bernoulli polynomials ${ }_{H} B_{n}^{(k)}(x, y ; a, b, e)$ are defined by

$$
\begin{aligned}
G_{3}(t, x, y ; k, a, b, e)= & \frac{\mathrm{Li}_{k}\left(1-(a b)^{-t}\right)}{b^{t}-a^{-t}} e^{x t+y t^{2}}=\sum_{n=0}^{\infty}{ }_{H} B_{n}^{(k)}(x, y ; a, b, e) \frac{t^{n}}{n !}, \\
& (|t|<2 \pi /(|\ln a+\ln b|), x, y \in \mathbb{R}) .
\end{aligned}
$$

The generalized Hermite poly-Bernoulli polynomials ${ }_{H} B_{n}^{(k)}(x, y ; a, b, c)$ defined by (2.2) have the following properties which are stated as theorems below.

Theorem 1. Let $a, b, c>0$ and $a \neq b$. For $x, y \in \mathbb{R}$ and $n \geq 0$, we have

$$
\begin{aligned}
{ }_{H} B_{n}^{(k)}(x, y ; e, 1, e) & ={ }_{H} B_{n}^{(k)}(x, y),{ }_{H} B_{n}^{(k)}(0,0 ; a, b, 1)=B_{n}^{(k)}(a, b) \\
{ }_{H} B_{n}^{(k)}(0,0 ; e, 1,1) & =B_{n}^{(k)},{ }_{H} B_{n}^{(k)}(0,0 ; a, b, e)={ }_{H} B_{n}^{(k)}(a, b), \\
{ }_{H} B_{n}^{(k)}(x+y, z+u ; a, b, c) & =\sum_{m=0}^{n}\left(\begin{array}{c}
n \\
m
\end{array}\right) H_{m}(y, z ; c){ }_{H} B_{n-m}^{(k)}(x, u ; a, b, c), \\
{ }_{H} B_{n}^{(k)}(x+z, y ; a, b, c) & =\sum_{m=0}^{n}\left(\begin{array}{c}
n \\
m
\end{array}\right) B_{n-m}^{(k)}(x ; a, b, c) H_{m}(y, z ; c) .
\end{aligned}
$$


Proof. The formula in (2.6) are obvious. Applying definition (2.2), we have

$$
\begin{gathered}
\sum_{n=0}^{\infty}{ }_{H} B_{n}^{(k)}(x+y, z+u ; a, b, c) \frac{t^{n}}{n !}=\sum_{n=0}^{\infty}{ }_{H} B_{n}^{(k)}(x, u ; a, b, c) \frac{t^{n}}{n !} \sum_{m=0}^{\infty} H_{m}(y, z ; c) \frac{t^{m}}{m !} \\
=\sum_{n=0}^{\infty} \sum_{m=0}^{n} H_{m}(y, z ; c)_{H} B_{n-m}^{(k)}(x, u ; a, b, c) \frac{t^{n}}{(n-m) ! m !} .
\end{gathered}
$$

Now equating the coefficients of the like powers of $t$ in the above equation, we get the result (2.7).

Again, by using (2.2) of generalized Hermite poly-Bernoulli polynomials, we have

$$
\frac{\mathrm{Li}_{k}\left(1-(a b)^{-t}\right)}{b^{t}-a^{-t}} c^{(x+z) t+y t^{2}}=\sum_{n=0}^{\infty}{ }_{H} B_{n}^{(k)}(x+z, y ; a, b, c) \frac{t^{n}}{n !},
$$

which can be written as

$$
\frac{\operatorname{Li}_{k}\left(1-(a b)^{-t}\right)}{b^{t}-a^{-t}} c^{x t} c^{z t+y t^{2}}=\sum_{n=0}^{\infty} B_{n}^{(k)}(x ; a, b, c) \frac{t^{n}}{n !} \sum_{m=0}^{\infty} H_{m}(y, z ; c) \frac{t^{m}}{m !} .
$$

Replacing $n$ by $n-m$ in (2.10) and comparing with (2.9) and equating their coefficients of $t^{n}$ leads to formula (2.8).

\section{IMPLICIT SUMMATION FORMUALE INVOLVING GENERALIZED HERMITE POLY-BERNOULLI POLYNOMIALS}

For the derivation of implicit formulae involving generalized poly-Bernoulli polynomials $B_{n}^{(k)}(x ; a, b, c)$ and generalized Hermite poly-Bernoulli polynomials ${ }_{H} B_{n}^{(k)}$ $(x, y ; a, b, c)$ the same considerations as developed for the ordinary Hermite and related polynomials in Khan [7-9] and Hermite-Bernoulli polynomials in Pathan and Khan [12-17] holds as well. First we prove the following results involving generalized Hermite poly-Bernoulli polynomials ${ }_{H} B_{n}^{(k)}(x, y ; a, b, c)$.

Theorem 2. Let $a, b, c>0$ and $a \neq b$. For $x, y \in \mathbb{R}$ and $n \geq 0$, the following implicit summation formulae for generalized Hermite poly-Bernoulli polynomials ${ }_{H} B_{n}^{(k)}(x, y ; a, b, c)$ holds true:

$$
\begin{aligned}
& { }_{H} B_{l+p}^{(k)}(z, y ; a, b, c) \\
& \quad=\sum_{m, n=0}^{l, p}\left(\begin{array}{l}
l \\
m
\end{array}\right)\left(\begin{array}{l}
p \\
n
\end{array}\right)(z-x)^{m+n}(\ln c)^{m+n}{ }_{H} B_{l+p-m-n}^{(k)}(x, y ; a, b, c) .
\end{aligned}
$$

Proof. We replace $t$ by $t+u$ and rewrite the generating function (2.2) as

$$
\frac{\operatorname{Li}_{k}\left(1-(a b)^{-(t+u)}\right)}{b^{t+u}-a^{-(t+u)}} c^{y(t+u)^{2}}=c^{-x(t+u)} \sum_{l, p=0}^{\infty}{ }_{H} B_{l+p}^{(k)}(x, y ; a, b, c) \frac{t^{l}}{l !} \frac{u^{p}}{p !} .
$$


Replacing $x$ by $z$ and equating the resulting equation to the above equation, we get

$$
c^{(z-x)(t+u)} \sum_{l, p=0}^{\infty}{ }_{H} B_{l+p}^{(k)}(x, y ; a, b, c) \frac{t^{l}}{l !} \frac{u^{p}}{p !}=\sum_{l, p=0}^{\infty}{ }_{H} B_{l+p}^{(k)}(z, y ; a, b, c) \frac{t^{l}}{l !} \frac{u^{p}}{p !} .
$$

On expanding exponential function (3.3) gives

$$
\begin{aligned}
\sum_{N=0}^{\infty} \frac{[(z-x)(t+u)]^{N}}{N !} \sum_{l, p=0}^{\infty}{ }_{H} B_{l+p}^{(k)}(x, y ; a, b, c) \frac{t^{l}}{l !} \frac{u^{p}}{p !} \\
=\sum_{l, p=0}^{\infty}{ }_{H} B_{l+p}^{(k)}(z, y ; a, b, c) \frac{t^{l}}{l !} \frac{u^{p}}{p !}
\end{aligned}
$$

which on using formula $[18, p .52(2)]$ :

$$
\sum_{N=0}^{\infty} f(N) \frac{(x+y)^{N}}{N !}=\sum_{n, m=0}^{\infty} f(n+m) \frac{x^{n}}{n !} \frac{y^{m}}{m !},
$$

in the left hand side becomes

$$
\begin{gathered}
\sum_{m, n=0}^{\infty} \frac{(z-x)^{m+n}(\ln c)^{m+n} t^{m} u^{n}}{m ! n !} \sum_{l, p=0}^{\infty}{ }_{H} B_{l+p}^{(k)}(x, y ; a, b, c) \frac{t^{l}}{l !} \frac{u^{p}}{p !} \\
\quad=\sum_{l, p=0}^{\infty}{ }_{H} B_{l+p}^{(k)}(z, y ; a, b, c) \frac{t^{l}}{l !} \frac{u^{p}}{p !} .
\end{gathered}
$$

Now replacing $l$ by $l-m, p$ by $p-n$ and using the lemma [18, p.100(1)] in the left hand side of (3.7), we get

$$
\begin{aligned}
\sum_{m, n=0}^{\infty} & \sum_{l, p=0}^{\infty} \frac{(z-x)^{m+n}(\ln c)^{m+n}}{m ! n !}{ }_{H} B_{l+p-m-n}^{(k)}(x, y ; a, b, c) \frac{t^{l}}{(l-m) !} \frac{u^{p}}{(p-n) !} \\
= & \sum_{l, p=0}^{\infty}{ }_{H} B_{l+p}^{(k)}(z, y ; a, b, c) \frac{t^{l}}{l !} \frac{u^{p}}{p !} .
\end{aligned}
$$

Finally, on equating the coefficients of the like powers of $t$ and $u$ in the above equation, we get the required result.

Remark 1 . On setting $l=0$ in Theorem 3.1, we immediately deduce the following result.

Corollary 1. The following implicit summation formula for Hermite poly-Bernoulli polynomials ${ }_{H} B_{n}^{(k)}(z, y ; a, b, c)$ holds true:

$$
{ }_{H} B_{p}^{(k)}(z, y ; a, b, c)=\sum_{n=0}^{p}\left(\begin{array}{c}
p \\
n
\end{array}\right)(z-x)^{n}(\ln c)^{n}{ }_{H} B_{p-n}^{(k)}(x, y ; a, b, c) .
$$


Remark 2. Replacing $z$ by $z+x$ and setting $y=0$ in Theorem 3.1, we obtain the following result involving generalized poly-Bernoulli polynomials of one variable

$$
B_{l+p}^{(k)}(z+x ; a, b, c)=\sum_{m, n=0}^{l, p}\left(\begin{array}{l}
l \\
m
\end{array}\right)\left(\begin{array}{l}
p \\
n
\end{array}\right)(z)^{m+n}(\ln c)^{m+n} B_{l+p-m-n}^{(k)}(x ; a, b, c),
$$

whereas by setting $z=0$ in Theorem 3.1, we obtain another result involving generalized poly-Bernoulli polynomials of one and two variables

$$
B_{l+p}^{(k)}(y ; a, b, c)=\sum_{m, n=0}^{l, p}\left(\begin{array}{l}
l \\
m
\end{array}\right)\left(\begin{array}{l}
p \\
n
\end{array}\right)(-x)^{m+n}(\ln c)^{m+n} B_{l+p-m-n}^{(k)}(x, y ; a, b, c) .
$$

Remark 3. Along with the above results we will exploit extended forms of generalized poly-Bernoulli polynomials $B_{l+p}^{(k)}(z ; a, b, c)$ by setting $y=0$ in the Theorem 3.1 to get

$$
B_{l+p}^{(k)}(z ; a, b, c)=\sum_{m, n=0}^{l, p}\left(\begin{array}{l}
l \\
m
\end{array}\right)\left(\begin{array}{l}
p \\
n
\end{array}\right)(z-x)^{n+m}(\ln c)^{m+n} B_{l+p-m-n}^{(k)}(x ; a, b, c) .
$$

Theorem 3. Let $a, b, c>0$ and $a \neq b$. Then $x \in \mathbb{R}$ and $n \geq 0$, we have

$$
B_{n}^{(k)}(x+1 ; a, b, c)=B_{n}^{(k)}\left(x ; a c, \frac{b}{c}, c\right) .
$$

Proof. From (2.3), we have

$$
\begin{aligned}
& \sum_{n=0}^{\infty} B_{n}^{(k)}(x+1 ; a, b, c) \frac{t^{n}}{n !}=\frac{\operatorname{Li}_{k}\left(1-(a b)^{-t}\right)}{b^{t}-a^{-t}} c^{(x+1) t}=\frac{\operatorname{Li}_{k}\left(1-(a b)^{-t}\right)}{b^{t}-a^{-t}} c^{x t} c^{t} \\
& \sum_{n=0}^{\infty} B_{n}^{(k)}(x+1 ; a, b, c) \frac{t^{n}}{n !}=\frac{\operatorname{Li}_{k}\left(1-(a b)^{-t}\right)}{\left(\frac{b}{c}\right)^{t}-(a c)^{-t}} c^{x t}=\sum_{n=0}^{\infty} B_{n}^{(k)}\left(x ; a c, \frac{b}{c}, c\right) \frac{t^{n}}{n !} .
\end{aligned}
$$

Equating the coefficients of $t^{n}$ on both sides, we get (3.13).

Theorem 4. Let $a, b, c>0$ and $a \neq b$. Then for $x, y \in \mathbb{R}$ and $n \geq 0$, we have

$$
{ }_{H} B_{n}^{(k)}(x+1, y ; a, b, c)=\sum_{j=0}^{\left[\frac{n}{2}\right]}\left(\begin{array}{l}
n \\
2 j
\end{array}\right) y^{j}(\ln c)^{j} B_{n-2 j}^{(k)}\left(x ; a c, \frac{b}{c}, c\right) .
$$

Proof. Since

$$
\begin{gathered}
{ }_{H} B_{n}^{(\alpha)}(x+1, y ; a, b, c) \frac{t^{n}}{n !}=\frac{\operatorname{Li}_{k}\left(1-(a b)^{-t}\right)}{b^{t}-a^{-t}} c^{(x+1) t+y t^{2}}=\frac{\operatorname{Li}_{k}\left(1-(a b)^{-t}\right)}{\left(\frac{b}{c}\right)^{t}-(a c)^{-t}} c^{x t} c^{y t^{2}} \\
=\left(\sum_{n=0}^{\infty} B_{n}^{(k)}\left(x ; a c, \frac{b}{c}, c\right) \frac{t^{n}}{n !}\right)\left(\sum_{j=0}^{\infty} y^{j}(\ln c)^{j} \frac{t^{2} j}{j !}\right) .
\end{gathered}
$$


Now replacing $n$ by $n-2 j$ and comparing the coefficients of $t^{n}$, we obtain the result (3.15).

Theorem 5. Let $a, b, c>0$ and $a \neq b$. Then for $x, y \in \mathbb{R}$ and $n \geq 0$, we have

$$
{ }_{H} B_{n}^{(k)}(x, y ; a, b, c)=\sum_{m=0}^{n}\left(\begin{array}{l}
n \\
m
\end{array}\right) B_{n-m}^{(k)}(a, b) H_{m}(x, y, c) .
$$

Proof. By using equations (2.2) and (1.2), we have

$$
\begin{aligned}
\frac{\operatorname{Li}_{k}\left(1-(a b)^{-t}\right)}{b^{t}-a^{-t}} c^{x t+y t^{2}} & =\sum_{n=0}^{\infty}{ }_{H} B_{n}^{(k)}(x, y ; a, b, c) \frac{t^{n}}{n !} \\
& =\left(\sum_{n=0}^{\infty} B_{n}^{(k)}(a, b) \frac{t^{n}}{n !}\right)\left(\sum_{m=0}^{\infty} H_{m}(x, y ; c) \frac{t^{m}}{m !}\right) .
\end{aligned}
$$

Replacing $n$ by $n-m$ and comparing the coefficients of $t^{n}$, we required at the desired result (3.16).

Remark 4. For $c=e$, (3.16) yields

$$
{ }_{H} B_{n}^{(k)}(x, y ; a, b, e)=\sum_{m=0}^{n}\left(\begin{array}{c}
n \\
m
\end{array}\right) B_{n-m}^{k}(a, b) H_{m}(x, y) .
$$

Theorem 6. Let $a, b, c>0$ and $a \neq b$. Then for $x, y \in \mathbb{R}$ and $n \geq 0$, we have

$$
{ }_{H} B_{n}^{(k)}(x, y ; a, b, c)=\sum_{m=0}^{n-2 j} \sum_{j=0}^{\left[\frac{n}{2}\right]} y^{j} x^{n-m-2 j}(\ln c)^{n-m-j} B_{m}^{(k)}(a, b) \frac{n !}{m ! j !(n-2 j-m) !} .
$$

Proof. Applying the definition (2.2) to the term $\frac{\operatorname{Li}_{k}\left(1-(a b)^{-t}\right)}{b^{t}-a^{-t}}$ and expanding the exponential function $c^{x t+y t^{2}}$ at $t=0$ yields

$$
\begin{gathered}
\frac{\operatorname{Li}_{k}\left(1-(a b)^{-t}\right)}{b^{t}-a^{-t}} c^{x t+y t^{2}}=\left(\sum_{m=0}^{\infty} B_{m}^{(k)}(a, b) \frac{t^{m}}{m !}\right)\left(\sum_{n=0}^{\infty} x^{n}(\ln c)^{n} \frac{t^{n}}{n !}\right)\left(\sum_{j=0}^{\infty} y^{j}(\ln c)^{j} \frac{t^{2 j}}{j !}\right) \\
=\sum_{n=0}^{\infty}\left(\sum_{m=0}^{n}\left(\begin{array}{c}
n \\
m
\end{array}\right)(\ln c)^{n-m} B_{m}^{(k)}(a, b) x^{n-m}\right) \frac{t^{n}}{n !}\left(\sum_{j=0}^{\infty} y^{j}(\ln c)^{j} \frac{t^{2 j}}{j !}\right) .
\end{gathered}
$$

Replacing $n$ by $n-2 j$ in the L.H.S. of above equation, we have

$$
\begin{aligned}
& \sum_{n=0}^{\infty}{ }_{H} B_{n}^{(k)}(x, y ; a, b) \frac{t^{n}}{n !} \\
& \quad=\sum_{n=0}^{\infty}\left(\sum_{m=0}^{n-2 j} \sum_{j=0}^{\left[\frac{n}{2}\right]}\left(\begin{array}{l}
n-2 j \\
m
\end{array}\right)(\ln c)^{n-m-j} B_{m}^{(k)}(a, b) x^{n-m-2 j} y^{j}\right) \frac{t^{n}}{(n-2 j) ! j !} .
\end{aligned}
$$

Combining (3.18) and (2.2) and equating their coefficients of $t^{n}$ produce the formula (3.17). 
Theorem 7. Let $a, b, c>0$ and $a \neq b$. Then for $x, y \in \mathbb{R}$ and $n \geq 0$, we have

$$
{ }_{H} B_{n}^{(k)}(x+1, y ; a, b, c)=\sum_{j=0}^{\left[\frac{n}{2}\right]} \sum_{m=0}^{n-2 j}\left(\begin{array}{l}
n-2 j \\
m
\end{array}\right) y^{j}(\ln c)^{n-m-j} B_{m}^{(k)}(x ; a, b, c) .
$$

Proof. By using the definition of generalized Hermite poly-Bernoulli polynomials, we have

$$
\begin{aligned}
\frac{\operatorname{Li}_{k}\left(1-(a b)^{-t}\right)}{b^{t}-a^{-t}} & c^{(x+1) t+y t^{2}}=\sum_{n=0}^{\infty}{ }_{H} B_{n}^{(k)}(x+1, y ; a, b, c) \frac{t^{n}}{n !}, \\
& =\left(\sum_{m=0}^{\infty} B_{m}^{(k)}(x ; a, b, c) \frac{t^{m}}{m !}\right)\left(\sum_{n=0}^{\infty}(\ln c)^{n} \frac{t^{n}}{n !}\right)\left(\sum_{j=0}^{\infty} y^{j}(\ln c)^{j} \frac{t^{2 j}}{j !}\right) \\
& =\sum_{n=0}^{\infty} \sum_{m=0}^{n}\left(\begin{array}{c}
n \\
m
\end{array}\right)(\ln c)^{n-m} B_{m}^{(k)}(x ; a, b, c) \frac{t^{n}}{n !}\left(\sum_{j=0}^{\infty} y^{j}(\ln c)^{j} \frac{t^{2 j}}{j !}\right) \\
& =\sum_{n=0}^{\infty} \sum_{j=0}^{\infty} \sum_{m=0}^{n}\left(\begin{array}{c}
n \\
m
\end{array}\right) y^{j}(\ln c)^{n-m+j} B_{m}^{(k)}(x ; a, b, c) \frac{t^{n+2 j}}{n ! j !} .
\end{aligned}
$$

Replacing $n$ by $n-2 j$ in the L.H.S. of above equation, we have

$$
\begin{aligned}
\sum_{n=0}^{\infty}{ }_{H} B_{n}^{(k)}(x+1, y ; a, b, c) \frac{t^{n}}{n !} \\
\quad=\sum_{n=0}^{\infty}\left(\sum_{j=0}^{\left[\frac{n}{2}\right]} \sum_{m=0}^{n-2 j}\left(\begin{array}{l}
n-2 j \\
m
\end{array}\right) y^{j}(\ln c)^{n-m-j} B_{m}^{(k)}(x ; a, b, c)\right) \frac{t^{n}}{n !} .
\end{aligned}
$$

Combining (3.20) and (3.21) and equating their coefficients of $t^{n}$ leads to formula (3.19).

Theorem 8. Let $a, b, c>0$ and $a \neq b$. Then for $x, y \in \mathbb{R}$ and $n \geq 0$, we have

$$
{ }_{H} B_{n}^{(k)}(x+1, y ; a, b, c)=\sum_{m=0}^{n}\left(\begin{array}{l}
n \\
m
\end{array}\right)(\ln c)^{n-m}{ }_{H} B_{m}^{(k)}(x, y ; a, b, c) .
$$

Proof. From (2.2), we have

$$
\begin{aligned}
\sum_{n=0}^{\infty}{ }_{H} B_{n}^{(k)} & (x+1, y ; a, b, c) \frac{t^{n}}{n !}-\sum_{n=0}^{\infty}{ }_{H} B_{n}^{(k)}(x, y ; a, b, c) \frac{t^{n}}{n !} \\
& =\frac{\operatorname{Li}_{k}\left(1-(a b)^{-t}\right)}{b^{t}-a^{-t}} c^{x t+y t^{2}}\left(c^{t}-1\right) \\
& =\left(\sum_{m=0}^{\infty}{ }_{H} B_{m}^{(k)}(x, y ; a, b, c) \frac{t^{m}}{m !}\right)\left(\sum_{n=0}^{\infty}(\ln c)^{n} \frac{t^{n}}{n !}\right)-\sum_{n=0}^{\infty}{ }_{H} B_{n}^{(k)}(x, y ; a, b, c) \frac{t^{n}}{n !} \\
& =\sum_{n=0}^{\infty} \sum_{m=0}^{n}(\ln c)^{n-m}{ }_{H} B_{m}^{(k)}(x, y ; a, b, c) \frac{t^{n}}{(n-m) !}-\sum_{n=0}^{\infty}{ }_{H} B_{n}^{(k)}(x, y ; a, b, c) \frac{t^{n}}{n !}
\end{aligned}
$$


Finally, equating the coefficients of the like powers of $t^{n}$, we get (3.22).

Theorem 9. Let $a, b, c>0$ and $a \neq b$. Then for $x, y \in \mathbb{R}$ and $n \geq 0$, we have

$$
\sum_{m=0}^{n}\left(\begin{array}{c}
n \\
m
\end{array}\right)(\ln a b)^{m}{ }_{H} B_{n-m}^{(k)}(-x, y ; a, b, c)=(-1)^{n}{ }_{H} B_{n}^{(k)}(x, y ; a, b, c) .
$$

Proof. We replace $t$ by $-t$ in (2.2) and then subtract the result from (2.2) itself finding

$$
c^{y t^{2}}\left[\frac{L i_{k}\left(1-(a b)^{-t}\right)}{b^{t}-a^{-t}}\left(c^{x t}-(a b)^{t} c^{-x t}\right)\right]=\sum_{n=0}^{\infty}\left[1-(-1)^{n}\right]_{H} B_{n}^{(k)}(x, y ; a, b, c) \frac{t^{n}}{n !},
$$

which is equivalent to

$$
\begin{aligned}
\sum_{n=0}^{\infty}{ }_{H} B_{n}^{(k)}(x, y ; a, b, c) \frac{t^{n}}{n !} & -\left(\sum_{m=0}^{\infty}(\ln a b)^{m} \frac{t^{m}}{m !}\right) \sum_{n=0}^{\infty}{ }_{H} B_{n}^{(k)}(-x, y ; a, b, c) \frac{t^{n}}{n !} \\
& =\sum_{n=0}^{\infty}\left[1-(-1)^{n}\right]_{H} B_{n}^{(k)}(x, y ; a, b, c) \frac{t^{n}}{n !} \\
\sum_{n=0}^{\infty}{ }_{H} B_{n}^{(k)}(x, y ; a, b, c) \frac{t^{n}}{n !} & -\left(\sum_{n=0}^{\infty} \sum_{m=0}^{n}(\ln a b)^{m}\right){ }_{H} B_{n-m}^{(k)}(-x, y ; a, b, c) \frac{t^{n}}{(n-m) ! m !} \\
& =\sum_{n=0}^{\infty}\left[1-(-1)^{n}\right]_{H} B_{n}^{(k)}(x, y ; a, b, c) \frac{t^{n}}{n !},
\end{aligned}
$$

and thus by equating coefficients of like powers of $t^{n}$, we get (3.23).

\section{SYMMETRY IDENTITIES}

In this section, we establish general symmetry identities for the generalized polyBernoulli polynomials $B_{n}^{(k)}(x ; a, b, c)$ and the generalized Hermite-poly-Bernoulli polynomials ${ }_{H} B_{n}^{(k)}(x, y ; a, b, c)$ by applying the generating function (1.4) and (2.2). The results extend some known identities of Khan [7-9], Pathan and Khan [12-17].

Theorem 10. Let $a, b, c>0$ and $a \neq b$. Then for $x, y \in \mathbb{R}$ and $n \geq 0$, the following identity holds true:

$$
\begin{aligned}
\sum_{m=0}^{n}\left(\begin{array}{c}
n \\
m
\end{array}\right) b^{m} a^{n-m}{ }_{H} B_{n-m}^{(k)}\left(b x, b^{2} y ; A, B, c\right)_{H} B_{m}^{(k)}\left(a x, a^{2} y ; A, B, c\right) \\
=\sum_{m=0}^{n}\left(\begin{array}{c}
n \\
m
\end{array}\right) a^{m} b^{n-m}{ }_{H} B_{n-m}^{(k)}\left(a x, a^{2} y ; A, B, c\right)_{H} B_{m}^{(k)}\left(b x, b^{2} y ; A, B, c\right) .
\end{aligned}
$$

Proof. Start with

$$
g(t)=\left(\frac{\left(\operatorname{Li}_{k}\left(1-(a b)^{-t}\right)\right)^{2}}{\left(B^{a t}-A^{-a t}\right)\left(B^{b t}-A^{-b t}\right)}\right) c^{a b x t+a^{2} b^{2} y t^{2}} .
$$


Then the expression for $g(t)$ is symmetric in $a$ and $b$ and we can expand $g(t)$ into series in two ways to obtain:

$$
\begin{aligned}
g(t) & =\sum_{n=0}^{\infty}{ }_{H} B_{n}^{(k)}\left(b x, b^{2} y ; A, B, c\right) \frac{(a t)^{n}}{n !} \sum_{m=0}^{\infty}{ }_{H} B_{m}^{(k)}\left(a x, a^{2} y ; A, B, c\right) \frac{(b t)^{m}}{m !} \\
& =\sum_{n=0}^{\infty}\left(\sum_{m=0}^{n}\left(\begin{array}{c}
n \\
m
\end{array}\right) a^{n-m} b^{m}{ }_{H} B_{n-m}^{(k)}\left(b x, b^{2} y ; A, B, c\right)_{H} B_{m}^{(k)}\left(a x, a^{2} y ; A, B, c\right)\right) \frac{t^{n}}{n !}
\end{aligned}
$$

On the similar lines, we can show that

$$
\begin{aligned}
g(t) & =\sum_{n=0}^{\infty}{ }_{H} B_{n}^{(k)}\left(a x, a^{2} y ; A, B, c\right) \frac{(b t)^{n}}{n !} \sum_{m=0}^{\infty}{ }_{H} B_{m}^{(k)}\left(b x, b^{2} y ; A, B, c\right) \frac{(a t)^{m}}{m !} \\
& =\sum_{n=0}^{\infty}\left(\sum_{m=0}^{n}\left(\begin{array}{c}
n \\
m
\end{array}\right) a^{m} b^{n-m}{ }_{H} B_{n-m}^{(k)}\left(a x, a^{2} y ; A, B, c\right)_{H} B_{m}^{(k)}\left(b x, b^{2} y ; A, B, c\right)\right) \frac{t^{n}}{n !} .
\end{aligned}
$$

Comparing the coefficients of $t^{n}$ on the right hand sides of the last two equations, we arrive at the desired result.

Remark 5. For $c=e$ in Theorem 4.1, we get

$$
\begin{aligned}
\sum_{m=0}^{n}\left(\begin{array}{c}
n \\
m
\end{array}\right) b^{m} a^{n-m}{ }_{H} B_{n-m}^{(k)}\left(b x, b^{2} y ; A, B, e\right)_{H} B_{m}^{(k)}\left(a x, a^{2} y ; A, B, e\right) \\
=\sum_{m=0}^{n}\left(\begin{array}{c}
n \\
m
\end{array}\right) a^{m} b^{n-m}{ }_{H} B_{n-m}^{(k)}\left(a x, a^{2} y ; A, B, e\right)_{H} B_{m}^{(k)}\left(b x, b^{2} y ; A, B, e\right) .
\end{aligned}
$$

Remark 6. By setting $b=1$ in Theorem 4.1, the following result reduces to

$$
\begin{aligned}
\sum_{m=0}^{n}\left(\begin{array}{c}
n \\
m
\end{array}\right) a^{n-m}{ }_{H} B_{n-m}^{(k)}(x, y ; A, B, c)_{H} B_{m}^{(k)}\left(a x, a^{2} y ; A, B, c\right) \\
=\sum_{m=0}^{n}\left(\begin{array}{c}
n \\
m
\end{array}\right) a_{H}^{m} B_{n-m}^{(k)}\left(a x, a^{2} y ; A, B, c\right)_{H} B_{m}^{(k)}(x, y ; A, B, c) .
\end{aligned}
$$

Theorem 11. Let $a, b, c>0$ and $a \neq b$. Then for $x, y \in \mathbb{R}$ and $n \geq 0$, the following identity holds true:

$$
\begin{aligned}
& \sum_{m=0}^{n}\left(\begin{array}{c}
n \\
m
\end{array}\right) \sum_{i=0}^{a-1} \sum_{j=0}^{b-1}{ }_{H} B_{n-m}^{(k)}\left(b x+\frac{b}{a} i+j, b^{2} z ; A, B, c\right) B_{m}^{(k)}(a y ; A, B, c) b^{m} a^{n-m} \\
& =\sum_{m=0}^{n}\left(\begin{array}{c}
n \\
m
\end{array}\right) \sum_{i=0}^{b-1} \sum_{j=0}^{a-1}{ }_{H} B_{n-m}^{(k)}\left(a x+\frac{a}{b} i+j, a^{2} z ; A, B, c\right) B_{m}^{(k)}(b y ; A, B, c) a^{m} b^{n-m}
\end{aligned}
$$

Proof. Let

$$
h(t)=\left(\frac{\left(\operatorname{Li}_{k}\left(1-(a b)^{-t}\right)\right)^{2}}{\left(B^{a t}-A^{-a t}\right)\left(B^{b t}-A^{-b t}\right)}\right) \frac{\left(c^{a b t}-1\right)^{2} c^{a b(x+y) t+a^{2} b^{2} z t^{2}}}{\left(c^{a t}-1\right)\left(c^{b t}-1\right)} .
$$




$$
\begin{aligned}
h(t)= & \left(\frac{\operatorname{Li}_{k}\left(1-(a b)^{-t}\right)}{\left(B^{a t}-A^{-a t}\right.}\right) c^{a b x t+a^{2} b^{2} z t^{2}}\left(\frac{c^{a b t}-1}{c^{b t}-1}\right) \\
& \times\left(\frac{\operatorname{Li}_{k}\left(1-(a b)^{-t}\right)}{B^{b t}-A^{-b t}}\right) c^{a b y t}\left(\frac{c^{a b t}-1}{c^{a t}-1}\right) \\
= & \left(\frac{\operatorname{Li}_{k}\left(1-(a b)^{-t}\right)}{\left(B^{a t}-A^{-a t}\right.}\right) c^{a b x t+a^{2} b^{2} z t^{2}} \sum_{i=0}^{a-1} c^{b t i}\left(\frac{\operatorname{Li}_{k}\left(1-(a b)^{-t}\right)}{B^{b t}-A^{-b t}}\right) c^{a b y t} \sum_{j=0}^{b-1} c^{a t j} \\
= & \left(\frac{\operatorname{Li}_{k}\left(1-(a b)^{-t}\right)}{B^{a t}-A^{-a t}}\right) c^{a^{2} b^{2} z t^{2}} \sum_{i=0}^{a-1} \sum_{j=0}^{b-1} c^{\left(b x+\frac{b}{a} i+j\right) a t} \sum_{m=0}^{\infty} B_{m}^{(k)}(a y ; A, B, c) \frac{(b t)^{m}}{m !} \\
= & \sum_{n=0}^{\infty} \sum_{i=0}^{a-1} \sum_{j=0}^{b-1} B_{n}^{(k)}\left(b x+\frac{b}{a} i+j, b^{2} z ; A, B, c\right) \frac{(a t)^{n}}{n !} \sum_{m=0}^{\infty} B_{m}^{(k)}(a y ; A, B, c) \frac{(b t)^{m}}{(m) !} \\
= & \sum_{n=0}^{\infty}\left(\sum_{m=0}^{n}\left(\begin{array}{c}
n \\
m
\end{array}\right) \sum_{i=0}^{a-1} \sum_{j=0}^{b-1}{ }_{H} B_{n-m}^{(k)}\left(b x+\frac{b}{a} i+j, b^{2} z ; A, B, c\right)\right. \\
& \left.\times B_{m}^{(k)}(a y ; A, B, c) b^{m} a^{n-m}\right) \frac{t^{n}}{n !} .
\end{aligned}
$$

On the other hand, we have

$$
\begin{aligned}
h(t)= & \sum_{n=0}^{\infty}\left(\sum_{m=0}^{n}\left(\begin{array}{c}
n \\
m
\end{array}\right) \sum_{i=0}^{b-1} \sum_{j=0}^{a-1}{ }_{H} B_{n-m}^{(k)}\left(a x+\frac{a}{b} i+j, a^{2} z ; A, B, c\right)\right. \\
& \left.\times B_{m}^{(k)}(b y ; A, B, c) a^{m} b^{n-m}\right) \frac{t^{n}}{n !} .
\end{aligned}
$$

Comparing the coefficients of $\frac{t^{n}}{n !}$ on the right hand sides of the last two equations, we arrive at the desired result.

\section{ACKNOWLEDGEMENT}

The first author M. A. Pathan would like to thank the Department of Science and Technology, Government of India, for the financial assistance for this work under project number SR/S4/MS:794/12.

\section{REFERENCES}

[1] E. T. Bell, "Exponential polynomials," Annals of Mathematics, pp. 258-277, 1934.

[2] G. Dattoli, S. Lorenzutta, and C. Cesarano, "Finite sums and generalized forms of bernoulli polynomials," Rendiconti di Matematica, vol. 19, pp. 385-391, 1999.

[3] R. Dere and Y. Simsek, "Bernoulli type polynomials on umbral algebra," Russian Journal of Mathematical Physics, vol. 22, pp. 1-5, 2015, doi: 10.1134/S106192081501001X.

[4] H. Jolany and R. Corcino, "Explicit formula for generalization of poly-bernoulli numbers and polynomials with $a, b, c$ parameters," Journal of Classical Analysis, vol. 6, pp. 119-135, 2015, doi: $10.7153 /$ jca-06-10. 
[5] H. Jolany, M. Darafsheh, and R. E. Alikelaye, "Generalizations of poly-bernoulli numbers and polynomials," Int. J. Math. Comb, vol. 2, pp. 7-14, 2010. [Online]. Available: http://fs.unm.edu/IJMC/GeneralizationsOfPolyBernoulliNumbers.pdf

[6] M. Kaneko, "Poly-bernoulli numbers," Journal de théorie des nombres de Bordeaux, vol. 9, no. 1, pp. 221-228, 1997.

[7] W. A. Khan, "A note on degenerate hermite poly-bernoulli numbers and polynomials," J. Class. Anal, vol. 8, no. 1, pp. 65-76, 2016, doi: 10.7153/jca-08-06.

[8] W. A. Khan, "A note on hermite-based poly-euler and multi poly-euler polynomials," Palestine J. Math, vol. 5, no. 1, pp. 17-26, 2016. [Online]. Available: https://pjm.ppu.edu/sites/default/files/ papers/PJM_JUNE_2017_14.pdf

[9] W. A. Khan, "Some properties of the generalized apostol type hermite-based polynomials," Kyungpook Math. J, vol. 55, pp. 597-614, 2015, doi: 10.5666/KMJ.2015.55.3.597.

[10] Q.-M. Luo, B.-N. Guo, F. Qi, and L. Debnath, "Generalizations of bernoulli numbers and polynomials," International Journal of Mathematics and Mathematical Sciences, vol. 2003, 2003, doi: 10.1155/S0161171203112070.

[11] L. Milne-Thomson, "Two classes of generalized polynomials," Proceedings of the London Mathematical Society, vol. 2, no. 1, pp. 514-522, 1933.

[12] M. A. Pathan, "Some implicit summation formulas and symmetric identities for the generalized hermite-euler polynomials," East West Math, vol. 16, no. 01, pp. 92-109, 2014. [Online]. Available: http://fces.ntt.edu.vn/index.php/ewm/article/download/64/64

[13] M. A. Pathan and W. A. Khan, "Some implicit summation formulas and symmetric identities for the generalized hermite based-polynomials," Acta Universitatis Apulensis, vol. 39, pp. 113-136, 2014, doi: 10.17114/j.aua.2014.39.11.

[14] M. A. Pathan and W. A. Khan, "A new class of generalized polynomials associated with hermite and bernoulli polynomials," Le Matematiche, vol. 70, no. 1, pp. 53-70, 2015. [Online]. Available: https://lematematiche.dmi.unict.it/index.php/lematematiche/article/view/1107/933

[15] M. A. Pathan and W. A. Khan, "Some implicit summation formulas and symmetric identities for the generalized hermite-bernoulli polynomials," Mediterranean Journal of Mathematics, vol. 12, no. 3, pp. 679-695, 2015, doi: 10.1007/s00009-014-0423-0.

[16] M. A. Pathan and W. A. Khan, "Some new classes of generalized hermite-based apostol-euler and apostol-genocchi polynomials," Fasciculi Mathematici, vol. 55, no. 1, pp. 153-170, 2015, doi: 10.1515/fascmath-2015-0020.

[17] M. A. Pathan and W. A. Khan, "A new class of generalized polynomials associated with hermite and euler polynomials," Mediterranean Journal of Mathematics, vol. 13, no. 3, pp. 913-928, 2016 , doi: 10.1007/s00009-015-0551-1.

[18] H. M. Srivastava and H. L. Manocha, "Treatise on generating functions.” John Wiley \& Sons, Inc., 605 Third Ave., New York, NY 10158, USA, 1984, 500, 1984.

Authors' addresses

M. A. Pathan

Centre for Mathematical and Statistical Sciences (CMSS), KFRI,, Peechi P.O., Thrissur, Kerala680653, India

E-mail address: mapathan@gmail.com

Waseem A. Khan

Department of Mathematics and Natural Sciences, Prince Mohammad Bin Fahd University, P.O Box 1664, Al Khobar 31952, Saudi Arabia

E-mail address: wkhan1@ pmu.edu.sa 